\title{
Looking for the high way in EGFR-positive non-small cell lung cancer through the evaluation of survival endpoints
}

\author{
Antonio Passaro, Filippo de Marinis \\ Division of Thoracic Oncology, IEO, European Institute of Oncology IRCCS, Milan, Italy \\ Correspondence to: Antonio Passaro, MD, PhD. Division of Thoracic Oncology, European Institute of Oncology-IEO, Via G. Ripamonti, $435-20141$ \\ Milan, Italy. Email: antonio.passaro@ieo.it. \\ Provenance: This is an invited article commissioned by the Section Editor Hengrui Liang (Department of Thoracic Surgery, Guangzhou Medical \\ University, Guangzhou, China). \\ Comment on: Mok TS, Cheng Y, Zhou X, et al. Improvement in Overall Survival in a Randomized Study That Compared Dacomitinib With Gefitinib \\ in Patients With Advanced Non-Small-Cell Lung Cancer and EGFR-Activating Mutations. J Clin Oncol 2018;36:2244-50.
}

Submitted May 29, 2019. Accepted for publication Jun 10, 2019.

doi: $10.21037 /$ tlcr.2019.06.04

View this article at: http://dx.doi.org/10.21037/tlcr.2019.06.04

The precision medicine era in non-small cell lung cancer (NSCLC) was born with the identification of EGFR mutations and the possibility to treat patients harbouring EGFR alterations with specific and targeted tyrosine kinase inhibitors (1-3). Thinking about this progress today it seems to look at the prehistory of lung cancer treatment, but only about 10 years have passed in which we revolutionized the survival of our patients (4).

Different randomized clinical trials (RCTs) evaluated the role of reversible first-generation of EGFR TKIs (erlotinib and gefitinib), showing a clear benefit in improving response rate and progression-free survival (PFS) compared with platinum-based chemotherapy in EGFR-positive NSCLC (4-9).

However, none of these trials showed a significant improvement in overall survival (OS), as well as secondgeneration EGFR TKI afatinib did not demonstrate significant differences in OS, evaluated in two different phases III trials, Lunx-Lung 3 and 6 (10,11). In addition, afatinib failed also to show an improvement in OS when compared with gefitinib in a phase IIb (Lux-Lung 7), the first head-to-head clinical trial comparing two different EGFR TKIs $(12,13)$. Dacomitinib, the other secondgeneration EGFR TKIs, was compared with gefitinib in the ARCHER 1050, a phase III randomized evaluating the role of these two TKIs in the first-line setting of the mixed population (Asian and Caucasian) with NSCLC harbouring common (Del19 or L858R) mutations (14).
In ARCHER 1050, patients with uncommon mutations and brain metastases $(\mathrm{BM})$ were excluded as per protocol. Results showed that dacomitinib significantly improved PFS (14.7 vs. 9.2 months, HR 0.59; $\mathrm{P}<0.0001)$ and an OS (34.1 vs. 26.8, HR 0.76, $\mathrm{P}=0.438$ ) compared with gefitinib (14). Although these results of OS about dacomitinib appear very interesting and clinically relevant, they were not able to fully satisfy clinical needs and expectations of thoracic oncologists comparing with those of osimertinib from the FLAURA trial (15).

The results of FLAURA trial, a randomized double-blind study comparing osimertinib, a third-generation EGFR TKs, with standard EGFR TKIs (gefitinib or erlotinib), succeed in the introduction of a new standard of care (SoC) for patients with EGFR mutations with or without brain metastases (BM) and reporting a very favourable and manageable safety profile, despite data about OS are still not mature. In this trial, median PFS was significantly longer for patients receiving osimertinib versus first-generation standard EGFR TKI (18.9 vs. 10.2 months; HR 0.46; 95\% CI, 0.37-0.57; $\mathrm{P}=0.001)$ (15).

The baton to FLAURA was exchanged by the immediate AURA 2 and 3 runners, a clinical trials in EGFR resistant NSCLC, harbouring T790M resistant mutations, progressing on a previous first- or second-generation TKIs $(16,17)$. Based on the activity of osimertinib in resistant EGFR-positive NSCLC, the expectation about its role in TKI naïve disease, was very high. 
Table 1 Driving factors for first-line treatment choice in EGFRpositive NSCLC

\begin{tabular}{lc}
\hline Endpoint & Score \\
\hline Progression-free survival (PFS) & +++++ \\
CNS activity & +++++ \\
Quality of Life (QoL) & ++++ \\
Rate of PD in the first 3 months of treatment & ++++ \\
Overall survival (OS) & +++ \\
Safety & +++ \\
Long-term toxicities & +++ \\
Rate of subsequent therapy & ++ \\
Response rate (RR) & ++ \\
Mechanisms of resistance & ++ \\
Cost-effectiveness & + \\
\hline
\end{tabular}

+ , with limited significance; ++, slightly significant; +++, moderately significant; ++++, very significant; +++++, extremely significant. NSCLC, non-small cell lung cancer.

Despite 18.9 months was never achieved before in front-line setting of NSCLC carrying common EGFR mutations, this results was initially accepted with a little bit of disappointment, becoming later source of clinical satisfaction.

The initial disappointment was related to the theoretical view that a mathematical sum of PFS1 (first or secondgeneration TKIs) plus PFS2 (osimertinib) is able to generate a survival higher of 20 months.

To better understand the role of treatment sequences, we have to considering that the EGFR T790M acquired mutation is developed in about $50 \%$ of tested patients that identifying the only $25-30 \%$ of overall EGFRpositive patient previously treated with the first- or secondgeneration TKIs $(18,19)$.

Following this wave, osimertinib acquired worldwide its dominant position becoming the new standard of care for treatment naïve patients with EGFR-mutations.

Clearly, these premises suggests that to identify the best first-line treatment in EGFR positive NSCLC, we should take into account different factors, that considered as a whole, they may be able to suggest the best way to follow improving clinical and survival outcomes (Table 1).

OS as main endpoint in targeted therapy of NSCLC remains ambiguous due to the heterogeneity of subsequent lines of therapy, which hold great methodological (for understanding the results of the studies) and clinical significance $(20,21)$.

The rates of subsequent therapies reported in RCTs of EGFR TKIs was $60-70 \%$ for first-generation EGFR TKIs, $71 \%$ for afatinib $(22,23)$ and only $49.8 \%$ for dacomitinib in the ARCHER 1050 trial, in which $9.7 \%$ for patients receiving dacomitinib and $11.1 \%$ for those previously treated with gefitinib received a third-generation EGFR TKIs (24). In the FLAURA trial, 59\% of patients that received osimertinib and $61 \%$ of those in the SoC EGFRTKI arm started a second-line therapy, that was platinumbased chemotherapy in the osimertinib arm, and osimertinib in the SoC EGFR (25).

Surely, the main question remains if the OS should be considered instead of PFS as principal survival endpoint to identify the best EGFR-TKIs. We know, that OS, defined as the time from randomization to death from any cause, is a direct and effective measure of clinical benefit to evaluate the survival of NSCLC patients in clinical trials, but its magnitude might be variably confounded by the activity of subsequent line of treatments, whose collection is not standardized in clinical trials, amenable to multiple condouders, as well. Of course, OS is easily measured, objective and clinically relevant survival endpoint but probably is not the best to use for clinical trials assessing new drugs in oncogene-addicted malignancies, differing from the immunotherapy clinical trials $(20,21,26,27)$.

These data suggests that the question about the role of OS remains still open, despite the non-binding recommendation of Food and Drug Administration (FDA) suggesting to consider the it as the standard clinical benefit endpoint that should be used to establish the efficacy of a treatment in patients with locally advanced or metastatic NSCLC $(20,21,26,27)$.

The question, therefore, for OS remains: what is clinically meaningful and what are most valuable tools at hand to measure it in EGFR-positive (oncogene addicted) NSCLC (28)?

Probably, considering the high chance-rate of multiple treatment lines after a first-line with EGFR TKI, the PFS should be considered as the most informative survival endpoint in clinical trials, particularly when the trial is not designed to assess the benefit of "sequences of treatments", that can cover great part of the survival of the patients in the metastatic settings $(20,27)$.

However, beyond the OS and PFS, the maintenance of disease control is critical in the first phase of treatments, namely in the first three months. It's highly significant, 
to guarantee an optimal disease control to the highest rate of patients, ensuring few early events of rapid disease progression. For instance, the clinical trials assessing alectinib of brigatinib compared with crizotinib in first-line setting of ALK rearranged NSCLC, suggested that using a second-generation TKIs upfront, is more effective reducing the rate of upfront resistant disease $(28,29)$.

Using a first or second-generation EGFR-TKIs (including dacomitinib) appear clearly less powerful than a third-generation TKI as osimertinib also shifting our attention the first months of treatment. Analyzing the KM curves of the ARCHER 1050 clinical trial, no differences between dacomitinib and gefitinib were reported in the first 3 and 6 months of treatment, with about $30 \%$ of progressive disease patients in both arms after 6 months of treatment. These data appear different from those of the FLAURA trial, in which only $6.1 \%(17 / 279)$ of patients that received osimertinib progressed during the first three months of treatment, compared with $13.7 \%$ of SoC $(38 / 277)$; data also confirmed considering at the cut-off of 6 months $(16.4 \%$ vs. $28.8 \%$ of progressive patients, in favour of osimertinib) Indeed, maximizing the rate of patients without progression in the first months of treatment should be considered of high importance choosing the most appropriate treatment options, balancing efficacy and safety that remaining a crucial point in view of long survival.

Moving one step forward over the evaluation of surrogate and not survival endpoints, to date the most important clinical factors significantly influencing the treatment choice and survival for the first-line setting in NSCLC carrying sensitive EGFR mutations is the central nervous system (CNS) involvement and the related activity of the different EGFR TKIs on BM (30,31). We know that the presence of $\mathrm{BM}$ is a crucial issue for the prognosis and quality of life (QoL) of patients with EGFR-positive NSCLC, considering a baseline incidence of about $25 / 30 \%$, and further risk of CNS progression of about $15-20 \%$ during EGFR TKIs treatment (31). Among patients with baseline pre-existing CNS involvements, the development of further BM is significantly more common and related with a significantly worse outcome, compared with those with no prior $\mathrm{BM}(2$ years cumulative incidence: $47 \%$ vs. $11 \% ; \mathrm{P}=0.003)(30)$.

Although preclinical and clinical evidence suggests that second- more than first-generation EGFR TKIs presented clinical activity in NSCLC with BM, these data are limited and not consistent compared with data about osimertinib. In patients with $\geq$ one measurable CNS lesion enrolled in the FLAURA trial, osimertinib showed a confirmed and considerable improved CNS-PFS (HR, 0.48, 95\% CI, 0.26-0.86; $\mathrm{P}=0.014)$ and intracranial ORR $(91 \%$ vs. 68\%) compared with first-generation TKIs (32).

CNS progression was a half with osimertinib compared with the standard EGFR-TKIs (20\% vs. 39\%), confirming a highly protective effect of osimertinib against BM. These data confirming the activity of osimertinib against brain involvement, are highly related to a significant improvement of QoL, reducing the possibilities of cancer-related symptoms and immediate or delayed toxicity of treatments (22).

Indeed, in the pre-osimertinib era, characterized by different EGFR TKIs with confirmed limited activity on CNS involvements, whole-brain radiotherapy (WBRT) and stereotactic radiosurgery (SRS) were the only ways to manage with momentary success CNS involvement due to NSCLC. Unfortunately, these different radiotherapy approaches are both associated with, immediately and lately side effects and may not improve survival and of sure accept on QoL (22).

Nevertheless, the issue of neurocognitive sequelae, although reduced in SRS compared to WBRT, is always to be considered particularly for patients with a longer life expectancy. In addition, the incidence of radionecrosis, steroid dependence and cognitive decline highlighted the important drawbacks of these methods, especially when compared to the activity and long-term safety of osimertinib in the same setting. The results of the CNS analysis of the FLAURA trial, confirmed that an upfront systemic therapy with osimertinib in patients with metastatic NSCLC harbouring sensitive EGFR mutations and BM should be considered the gold standard. This approach seems to be able to improve QoL, delaying radiotherapy that could be used at a later stage, when an adjunctive cerebral disease control may optimize the strategy of care, saving ammos in the case (22).

For an accurate therapeutic definition for the first line of the EGFR-positive NSCLC, it is needed that all the decision-making drivers at our disposal are taken into consideration and weighted. Based on all these premises and evaluations and although the OS results achieved by dacomitinib, can we consider it as the new treatment standard for the first line of EGFR-positive NSCLC, according to the survival gain alone? To be honest, most 
likely not, not today: perhaps less than 5 years ago, it would have dominated the clinical practice as an outbreaking novelty, no doubt!

Looking at dynamic landscape of drug development in NSCLC harbouring sensitive EGFR mutations, should be highly important to consider the discussed driving factors in the therapeutic decision, also to globally evaluate the upcoming results of new combination of EGFR TKIs with anti-VEGF (e.g., bevacizumab or ramucirumab) nowadays under investigation in different clinical trials.

\section{Acknowledgments}

None.

\section{Footnote}

Conflicts of Interest: A Passaro served as consultant/ advisory role for Astra Zeneca, Bristol Myers Squibb, Roche Genentech, Dako/Agilent, Merck Sharp \& Dome. F de Marinis served as consultant/advisory role for Astra Zeneca, Boeringher Inghleim, Bristol Myers Squibb, Roche Genentech, Merck Sharm \& Dome, Pfizer and Takeda.

Ethical Statement: The authors are accountable for all aspects of the work in ensuring that questions related to the accuracy or integrity of any part of the work are appropriately investigated and resolved.

\section{References}

1. Lynch TJ, Bell DW, Sordella R, et al. Activating mutations in the epidermal growth factor receptor underlying responsiveness of non-small-cell lung cancer to gefitinib. N Engl J Med 2004;350:2129-39.

2. Paez JG, Janne PA, Lee JC, et al. EGFR mutations in lung cancer: correlation with clinical response to gefitinib therapy. Science 2004;304:1497-500.

3. Kosaka T, Yatabe Y, Endoh H, et al. Mutations of the epidermal growth factor receptor gene in lung cancer: biological and clinical implications. Cancer Res 2004;64:8919-23.

4. Mok TS, Wu YL, Thongprasert S, et al. Gefitinib or carboplatin-paclitaxel in pulmonary adenocarcinoma. $\mathrm{N}$ Engl J Med 2009;361:947-57.

5. Rosell R, Carcereny E, Gervais R, et al. Erlotinib versus standard chemotherapy as first-line treatment for European patients with advanced EGFR mutation-positive non-small-cell lung cancer (EURTAC): a multicentre, open-label, randomised phase 3 trial. Lancet Oncol 2012;13:239-46.

6. Zhou C, Wu YL, Chen G, et al. Erlotinib versus chemotherapy as first-line treatment for patients with advanced EGFR mutation-positive non-small-cell lung cancer (OPTIMAL, CTONG-0802): a multicentre, open-label, randomised, phase 3 study. Lancet Oncol 2011;12:735-42.

7. Inoue A, Kobayashi K, Maemondo M, et al. Final overall survival results of NEJ002, a phase III trial comparing gefitinib to carboplatin (CBDCA) plus paclitaxel (TXL) as the first-line treatment for advanced non-small cell lung cancer (NSCLC) with EGFR mutations. J Clin Oncol 2011;29:abstr 7519.

8. Han JY, Park K, Kim SW, et al. First-SIGNAL: first-line single-agent iressa versus gemcitabine and cisplatin trial in never-smokers with adenocarcinoma of the lung. J Clin Oncol 2012;30:1122-8.

9. Mitsudomi T, Morita S, Yatabe Y, et al. Gefitinib versus cisplatin plus docetaxel in patients with non-small-cell lung cancer harbouring mutations of the epidermal growth factor receptor (WJTOG3405): an open label, randomised phase 3 trial. Lancet Oncol 2010;11:121-8.

10. Sequist LV, Yang JC, Yamamoto N, et al. Phase III study of afatinib or cisplatin plus pemetrexed in patients with metastatic lung adenocarcinoma with EGFR mutations. J Clin Oncol 2013;31:3327-34.

11. Wu YL, Zhou C, Hu CP, et al. Afatinib versus cisplatin plus gemcitabine for first-line treatment of Asian patients with advanced non-small-cell lung cancer harbouring EGFR mutations (LUX-Lung 6): an open-label, randomised phase 3 trial. Lancet Oncol 2014;15:213-22.

12. Yang JC, Hirsh V, Schuler M, et al. Symptom control and quality of life in LUX-Lung 3: a phase III study of afatinib or cisplatin/pemetrexed in patients with advanced lung adenocarcinoma with EGFR mutations. J Clin Oncol 2013;31:3342-50.

13. Passaro A, Pochesci A, Spitaleri G, et al. Afatinib in firstline setting for NSCLC harbouring common EGFR mutations: new light after the preliminary results of LUXLung 7? J Thorac Dis 2016;8:E217-20.

14. Wu YL, Cheng Y, Zhou X, et al. Dacomitinib versus gefitinib as first-line treatment for patients with EGFRmutation-positive non-small-cell lung cancer (ARCHER 1050): a randomised, open-label, phase 3 trial. Lancet Oncol 2017;18:1454-66. 
15. Soria JC, Ohe Y, Vansteenkiste J, et al. Osimertinib in untreated EGFR-mutated advanced non-small-cell lung cancer. N Engl J Med 2018;378:113-25.

16. Yang JC, Ahn MJ, Kim DW, et al. Osimertinib in pretreated T790M-positive advanced non-small-cell lung cancer: AURA study Phase II extension component. J Clin Oncol 2017;35:1288-96.

17. Mok TS, Wu YL, Ahn MJ, et al. Osimertinib or platinumpemetrexed in EGFR T790M-positive lung cancer. N Engl J Med 2017;376:629-40.

18. Fogli S, Polini B, Del Re M, et al. EGFR_TKIs in nonsmall cell lung cancer: focus on clinical pharmacology and mechanisms of resistance. Pharmacogenomics 2018;19:727-40.

19. Passaro, A, Gerini-Rocco E, Pochesci A, et al. Targeting EGFR T790M mutation in NSCLC: from biology to evaluation and treatment. Pharmacol Res 2017;117:406-15.

20. Fenchel K, Sellmann L, Dempke WC. Overall survival in non-small cell lung cancer-what is clinically meaningful? Transl Lung Cancer Res 2016;5:115-9.

21. Fenchel K, Dale SP, Dempke WC. Improved overall survival following tyrosine kinase inhibitor (TKI) treatment in NSCLC—are we making progress? Transl Lung Cancer Res 2016;5:373-6.

22. Girard N. Optimizing outcomes in EGFR mutationpositive NSCLC: which tyrosine kinase inhibitor and when? Future Oncol 2018;14:1117-32.

23. Sequist L, Wu Y, Schuler M, et al. Subsequent therapies post-afatinib among patients with EGFR mutationpositive NSCLC in LUX-Lung (LL) 3, 6 and 7. Ann Oncol 2017;28:v460-v496.

24. Mok TS, Cheng Y, Zhou X, et al. Improvement in Overall Survival in a Randomized Study That Compared Dacomitinib With Gefitinib in Patients With Advanced Non-Small-Cell Lung Cancer and EGFR-Activating

Cite this article as: Passaro A, de Marinis F. Looking for the high way in EGFR-positive non-small cell lung cancer through the evaluation of survival endpoints. Transl Lung Cancer Res 2019;8(Suppl 4):S334-S338. doi: 10.21037/tlcr.2019.06.04
Mutations. J Clin Oncol 2018;36:2244-50.

25. Planchard D, Boyer MJ, Lee JS, et al. Postprogression Outcomes for Osimertinib versus Standard-of-Care EGFR-TKI in Patients with Previously Untreated EGFRmutated Advanced Non-Small Cell Lung Cancer. Clin Cancer Res 2019;25:2058-63.

26. Pilz LR, Manegold C, Schid-Bindert G. Statistical considerations and endpoints for clinical lung cancer studies: can progression free survival (PFS) substitute overall survival (OS) as a valid endpoint in clinical trials for advanced non-small-cell lung cancer? Transl Lung Cancer Res 2012;1:26-35.

27. Clarke JM, Wang X, Ready NE. Surrogate clinical endpoints to predict overall survival in non-small cell lung cancer trials-are we in a new era? Transl Lung Cancer Res 2015;4:804-8.

28. Peters S, Camidge DR, Shaw AT, et al. Alectinib versus Crizotinib in Untreated ALK-Positive Non-Small-Cell Lung Cancer. N Engl J Med 2017;377:829-38.

29. Camidge DR, Kim HR, Ahn MJ, et al. Brigatinib versus Crizotinib in ALK-Positive Non-Small-Cell Lung Cancer. N Engl J Med 2018;379:2027-39.

30. Remon J, Besse B. Brain metastases in Oncogene-addicted Non-small cell lung cancer patients: incidence and treatment. Front Oncol 2018;8:88.

31. Passaro A, Gianoncelli L, Stati V, et al. Brain metastases in EGFR-positive non-small cell lung cancer: the way to the sanctuary becomes less winding. Ann Transl Med 2019;7:S80.

32. Reungwetwattana T, Nakagawa K, Cho BC, et al. CNS Response to Osimertinib Versus Standard Epidermal Growth Factor Receptor Tyrosine Kinase Inhibitors in Patients With Untreated EGFR-Mutated Advanced Non-Small-Cell Lung Cancer. J Clin Oncol 2018:JCO2018783118. [Epub ahead of print]. 